\title{
Congruences for the coefficients of the Gordon and McIntosh mock theta function $\xi(q)$
}

\author{
Robson da Silva and James A. Sellers
}

\begin{abstract}
Recently Gordon and McIntosh introduced the third order mock theta function $\xi(q)$ defined by

$$
\xi(q)=1+2 \sum_{n=1}^{\infty} \frac{q^{6 n^{2}-6 n+1}}{\left(q ; q^{6}\right)_{n}\left(q^{5} ; q^{6}\right)_{n}} .
$$

Our goal in this paper is to study arithmetic properties of the coefficients of this function. We present a number of such properties, including several infinite families of Ramanujan-like congruences.
\end{abstract}

Keywords: congruence, generating function, mock theta function Mathematics Subject Classification 2010: 11P83, 05A17

\section{Introduction}

In his last letter to Hardy in 1920, Ramanujan introduced the notion of a mock theta function. He listed 17 such functions having orders 3, 5, and 7 . Since then, other mock theta functions have been found. Gordon and McIntosh [7], for example, introduced many additional such functions, including the following of order 3 :

$$
\xi(q)=1+2 \sum_{n=1}^{\infty} \frac{q^{6 n^{2}-6 n+1}}{\left(q ; q^{6}\right)_{n}\left(q^{5} ; q^{6}\right)_{n}},
$$

where we use the standard $q$-series notation:

$$
\begin{aligned}
(a ; q)_{0} & =1 \\
(a ; q)_{n} & =(1-a)(1-a q) \cdots\left(1-a q^{n-1}\right), \forall n \geq 1, \\
(a ; q)_{\infty} & =\lim _{n \rightarrow \infty}(a ; q)_{n},|q|<1 .
\end{aligned}
$$


Arithmetic properties of the coefficients of mock theta functions have received a great deal of attention. For instance, Zhang and Shi [15] recently proved seven congruences satisfied by the coefficients of the mock theta function $\beta(q)$ introduced by McIntosh. In a recent paper, Brietzke, da Silva, and Sellers [5] found a number of arithmetic properties satisfied by the coefficients of the mock theta function $V_{0}(q)$, introduced by Gordon and McIntosh [6]. Andrews et al. [2] prove a number of congruences for the partition functions $p_{\omega}(n)$ and $p_{\nu}(n)$, introduced in [1], associated with the third order mock theta functions $\omega(q)$ and $\nu(q)$, where $\omega(q)$ is defined below and

$$
\nu(q)=\sum_{n=0}^{\infty} \frac{q^{n(n+1)}}{\left(-q ; q^{2}\right)_{n+1}} .
$$

In a subsequent paper, Wang [14] presented some additional congruences for both $p_{\omega}(n)$ and $p_{\nu}(n)$.

This paper is devoted to exploring arithmetic properties of the coefficients $p_{\xi}(n)$ defined by

$$
\sum_{n=0}^{\infty} p_{\xi}(n) q^{n}=\xi(q)
$$

It is clear from $(1)$ that $p_{\xi}(n)$ is even for all $n \geq 1$. In Sections 4 and 5 , we present other arithmetic properties of $p_{\xi}(n)$, including some infinite families of congruences.

\section{Preliminaries}

McIntosh [12, Theorem 3] proved a number of mock theta conjectures, including

$$
\begin{aligned}
& \omega(q)=g_{3}\left(q, q^{2}\right) \quad \text { and } \\
& \xi(q)=q^{2} g_{3}\left(q^{3}, q^{6}\right)+\frac{\left(q^{2} ; q^{2}\right)_{\infty}^{4}}{(q ; q)_{\infty}^{2}\left(q^{6} ; q^{6}\right)_{\infty}}
\end{aligned}
$$

where

$$
g_{3}(a, q)=\sum_{n=0}^{\infty} \frac{(-q ; q)_{n} q^{n(n+1) / 2}}{(a ; q)_{n+1}\left(a^{-1} q ; q\right)_{n+1}}
$$


and $\omega(q)$ is the third order mock theta functions given by

$$
\omega(q)=\sum_{n=0}^{\infty} \frac{q^{2 n(n+1)}}{\left(q ; q^{2}\right)_{n+1}^{2}} .
$$

It follows from (1), (3), and (4) that

$$
\xi(q)=q^{2} \omega\left(q^{3}\right)+\frac{\left(q^{2} ; q^{2}\right)_{\infty}^{4}}{(q ; q)_{\infty}^{2}\left(q^{6} ; q^{6}\right)_{\infty}} .
$$

Throughout the remainder of this paper, we define

$$
f_{k}:=\left(q^{k} ; q^{k}\right)_{\infty}
$$

in order to shorten the notation. Combining (5) and (2), we have

$$
\sum_{n=0}^{\infty} p_{\xi}(n) q^{n}=q^{2} \omega\left(q^{3}\right)+\frac{f_{2}^{4}}{f_{1}^{2} f_{6}} .
$$

We recall Ramanujan's theta functions

$$
\begin{aligned}
f(a, b) & :=\sum_{n=-\infty}^{\infty} a^{\frac{n(n+1)}{2}} b^{\frac{n(n-1)}{2}}, \text { for }|a b|<1, \\
\phi(q) & :=f(q, q)=\sum_{n=-\infty}^{\infty} q^{n^{2}}=\frac{f_{2}^{5}}{f_{1}^{2} f_{4}^{2}}, \text { and } \\
\psi(q) & :=f\left(q, q^{3}\right)=\sum_{n=0}^{\infty} q^{n(n+1) / 2}=\frac{f_{2}^{2}}{f_{1}} .
\end{aligned}
$$

The function $\phi(q)$ satisfies many identities, including (see [3, (22.4)])

$$
\phi(-q)=\frac{f_{1}^{2}}{f_{2}}
$$

In some of the proofs, we employ the classical Jacobi's identity (see [4, Theorem 1.3.9])

$$
f_{1}^{3}=\sum_{n=0}^{\infty}(-1)^{n}(2 n+1) q^{n(n+1) / 2} .
$$

We note the following identities which will be used below. 
Lemma 2.1. The following 2-dissection identities hold.

$$
\begin{aligned}
\frac{1}{f_{1}^{2}} & =\frac{f_{8}^{5}}{f_{2}^{5} f_{16}^{2}}+2 q \frac{f_{4}^{2} f_{16}^{2}}{f_{2}^{5} f_{8}}, \\
f_{1}^{2} & =\frac{f_{2} f_{8}^{5}}{f_{4}^{2} f_{16}^{2}}-2 q \frac{f_{2} f_{16}^{2}}{f_{8}} \\
\frac{1}{f_{1}^{4}} & =\frac{f_{4}^{14}}{f_{2}^{14} f_{8}^{4}}+4 q \frac{f_{4}^{2} f_{8}^{4}}{f_{2}^{10}}, \\
\frac{f_{3}}{f_{1}} & =\frac{f_{4} f_{6} f_{16} f_{24}^{2}}{f_{2}^{2} f_{8} f_{12} f_{48}}+q \frac{f_{6} f_{8}^{2} f_{48}}{f_{2}^{2} f_{16} f_{24}}, \\
\frac{f_{3}^{2}}{f_{1}^{2}} & =\frac{f_{4}^{4} f_{6} f_{12}^{2}}{f_{2}^{5} f_{8} f_{24}}+2 q \frac{f_{4} f_{6}^{2} f_{8} f_{24}}{f_{2}^{4} f_{12}}, \\
\frac{f_{1}^{3}}{f_{3}} & =\frac{f_{4}^{3}}{f_{12}}-3 q \frac{f_{2}^{2} f_{12}^{3}}{f_{4} f_{6}^{2}} \\
\frac{f_{3}}{f_{1}^{3}} & =\frac{f_{4}^{6} f_{6}^{3}}{f_{2}^{9} f_{12}^{2}}+3 q \frac{f_{4}^{2} f_{6} f_{12}^{2}}{f_{2}^{7}}, \\
\frac{1}{f_{1} f_{3}} & =\frac{f_{8}^{2} f_{12}^{5}}{f_{2}^{2} f_{4} f_{6}^{4} f_{24}^{2}}+q \frac{f_{4}^{5} f_{24}^{2}}{f_{2}^{4} f_{6}^{2} f_{8}^{2} f_{12}}
\end{aligned}
$$

Proof. By Entry 25 (i), (ii), (v), and (vi) in [3, p. 40], we have

$$
\begin{gathered}
\phi(q)=\phi\left(q^{4}\right)+2 q \psi\left(q^{8}\right), \\
\phi(q)^{2}=\phi\left(q^{2}\right)^{2}+4 q \psi\left(q^{4}\right)^{2} .
\end{gathered}
$$

Using (7) and (8) we can rewrite (19) in the form

$$
\frac{f_{2}^{5}}{f_{1}^{2} f_{4}^{2}}=\frac{f_{8}^{5}}{f_{4}^{2} f_{16}^{2}}+2 q \frac{f_{16}^{2}}{f_{8}}
$$

from which we obtain (11) after multiplying both sides by $\frac{f_{4}^{2}}{f_{5}^{5}}$. Identity (12) can be easily deduced from (11) using the procedure described in Section 30.10 of [9].

By (7) and (8) we can rewrite (3) in the form

$$
\frac{f_{2}^{10}}{f_{1}^{4} f_{4}^{4}}=\frac{f_{4}^{10}}{f_{2}^{4} f_{8}^{4}}+4 q \frac{f_{8}^{4}}{f_{4}^{2}}
$$

from which we obtain (13).

Identities (14), (15), and (18) are equations (30.10.3), (30.9.9), and (30.12.3) of [9], respectively. Finally, for proofs of (16) and (17) see [13, Lemma 4]. 
The next lemma exhibits the 3 -dissections of $\psi(q)$ and $1 / \phi(-q)$.

Lemma 2.2. We have

$$
\begin{aligned}
\psi(q) & =\frac{f_{6} f_{9}^{2}}{f_{3} f_{18}}+q \frac{f_{18}^{2}}{f_{9}} \\
\frac{1}{\phi(-q)} & =\frac{f_{6}^{4} f_{9}^{6}}{f_{3}^{8} f_{18}^{3}}+2 q \frac{f_{6}^{3} f_{9}^{3}}{f_{3}^{7}}+4 q^{2} \frac{f_{6}^{2} f_{18}^{3}}{f_{3}^{6}} .
\end{aligned}
$$

Proof. Identity (21) is equation (14.3.3) of [9]. A proof of (22) can be seen in [11].

\section{$3 \quad$ Dissections for $p_{\xi}(n)$}

This section is devoted to proving the 2-, 3-, and 4-dissections of (2). We begin with the 2-dissection.

Theorem 3.1. We have

$$
\begin{aligned}
2 \sum_{n=0}^{\infty} p_{\xi}(2 n+1) q^{n+1} & =\frac{f_{6}^{6} f_{12}}{f_{3}^{4} f_{24}^{2}}-f\left(q^{12}\right)+4 q \frac{f_{2}^{2} f_{8}^{2}}{f_{1} f_{3} f_{4}}, \quad \text { and } \\
\sum_{n=0}^{\infty} p_{\xi}(2 n) q^{n} & =q \frac{f_{6}^{8} f_{24}^{2}}{f_{3}^{4} f_{12}^{5}}-q^{4} \omega\left(-q^{6}\right)+\frac{f_{4}^{5}}{f_{1} f_{3} f_{8}^{2}} .
\end{aligned}
$$

Proof. We start with equation (4) of [2]:

$$
f\left(q^{8}\right)+2 q \omega(q)+2 q^{3} \omega\left(-q^{4}\right)=F(q),
$$

where $f(q)$ is the mock theta function

$$
f(q)=\sum_{n=0}^{\infty} \frac{q^{n^{2}}}{(-q ; q)_{n}^{2}}
$$

and

$$
F(q)=\frac{\phi(q) \phi\left(q^{2}\right)^{2}}{f_{4}^{2}}=\frac{f_{2} f_{6}^{6}}{f_{1}^{2} f_{12}^{4}}
$$

Thus,

$$
f\left(q^{24}\right)+2 q^{3} \omega\left(q^{3}\right)+2 q^{9} \omega\left(-q^{12}\right)=F\left(q^{3}\right) .
$$


Using (5), it follows that

$$
2 \sum_{n=0}^{\infty} p_{\xi}(n) q^{n+1}=F\left(q^{3}\right)-f\left(q^{24}\right)-2 q^{9} \omega\left(-q^{12}\right)+2 q \frac{f_{2}^{4}}{f_{1}^{2} f_{6}} .
$$

By (11), we have

$$
F\left(q^{3}\right)=\frac{f_{12}^{6} f_{24}}{f_{6}^{4} f_{48}^{2}}+2 q^{3} \frac{f_{12}^{8} f_{48}^{2}}{f_{6}^{4} f_{24}^{5}},
$$

which along with (11) allows us to rewrite (25) as

$$
\begin{aligned}
2 \sum_{n=0}^{\infty} p_{\xi}(n) q^{n+1}= & \frac{f_{12}^{6} f_{24}}{f_{6}^{4} f_{48}^{2}}+2 q^{3} \frac{f_{12}^{8} f_{48}^{2}}{f_{6}^{4} f_{24}^{5}}-f\left(q^{24}\right)-2 q^{9} \omega\left(-q^{12}\right) \\
& +2 q \frac{f_{8}^{5}}{f_{2} f_{6} f_{16}^{2}}+4 q^{2} \frac{f_{4}^{2} f_{16}^{2}}{f_{2} f_{6} f_{8}} .
\end{aligned}
$$

Thus,

$$
\begin{aligned}
2 \sum_{n=0}^{\infty} p_{\xi}(2 n+1) q^{2 n+2} & =\frac{f_{12}^{6} f_{24}}{f_{6}^{4} f_{48}^{2}}-f\left(q^{24}\right)+4 q^{2} \frac{f_{4}^{2} f_{16}^{2}}{f_{2} f_{6} f_{8}}, \quad \text { and } \\
\sum_{n=0}^{\infty} p_{\xi}(2 n) q^{2 n+1} & =q^{3} \frac{f_{12}^{8} f_{48}^{2}}{f_{6}^{4} f_{24}^{5}}-q^{9} \omega\left(-q^{12}\right)+q \frac{f_{8}^{5}}{f_{2} f_{6} f_{16}^{2}} .
\end{aligned}
$$

Dividing (27) by $q$ and replacing $q^{2}$ by $q$ in the resulting identity and in (26), we obtain (23) and (24).

The next theorem exhibits the 3-dissection of (2).

Theorem 3.2. We have

$$
\begin{aligned}
\sum_{n=0}^{\infty} p_{\xi}(3 n) q^{n} & =\frac{f_{2} f_{3}^{4}}{f_{1}^{2} f_{6}^{2}}, \\
\sum_{n=0}^{\infty} p_{\xi}(3 n+1) q^{n} & =2 \frac{f_{3} f_{6}}{f_{1}}, \text { and } \\
\sum_{n=0}^{\infty} p_{\xi}(3 n+2) q^{n} & =\omega(q)+\frac{f_{6}^{4}}{f_{2} f_{3}^{2}} .
\end{aligned}
$$


Proof. In view of (8), we rewrite (6) as

$$
\sum_{n=0}^{\infty} p_{\xi}(n) q^{n}=q^{2} \omega\left(q^{3}\right)+\frac{\psi(q)^{2}}{f_{6}} .
$$

Using (21), we obtain

$$
\sum_{n=0}^{\infty} p_{\xi}(n) q^{n}=q^{2} \omega\left(q^{3}\right)+\frac{f_{6} f_{9}^{4}}{f_{3}^{2} f_{18}^{2}}+2 q \frac{f_{9} f_{18}}{f_{3}}+q^{2} \frac{f_{18}^{4}}{f_{6} f_{9}^{2}} .
$$

Extracting the terms of the form $q^{3 n+r}$ on both sides of (31), for $r \in\{0,1,2\}$, dividing both sides of the resulting identity by $q^{r}$ and then replacing $q^{3}$ by $q$, we obtain the desired results.

We close this section with the 4-dissection of (2).

Theorem 3.3. We have

$$
\begin{aligned}
\sum_{n=0}^{\infty} p_{\xi}(4 n) q^{n} & =4 q^{2} \frac{f_{12}^{6}}{f_{3}^{2} f_{6}^{3}}-q^{2} \omega\left(-q^{3}\right)+\frac{f_{2}^{4} f_{6}^{5}}{f_{1}^{2} f_{3}^{4} f_{12}^{2}}, \\
\sum_{n=0}^{\infty} p_{\xi}(4 n+1) q^{n} & =2 q \frac{f_{6}^{3} f_{12}^{2}}{f_{3}^{4}}+2 \frac{f_{4}^{4} f_{6}^{5}}{f_{2}^{2} f_{3}^{4} f_{12}^{2}}, \\
\sum_{n=0}^{\infty} p_{\xi}(4 n+2) q^{n} & =\frac{f_{6}^{9}}{f_{3}^{6} f_{12}^{2}}+\frac{f_{2}^{10} f_{12}^{2}}{f_{1}^{4} f_{3}^{2} f_{4}^{4} f_{6}}, \quad \text { and } \\
2 \sum_{n=0}^{\infty} p_{\xi}(4 n+3) q^{n+1} & =\frac{f_{6}^{15}}{f_{3}^{8} f_{12}^{6}}-f\left(q^{6}\right)+4 q \frac{f_{2}^{4} f_{12}^{2}}{f_{1}^{2} f_{3}^{2} f_{6}} .
\end{aligned}
$$

Proof. In order to prove (32), we use (11) and (18) to obtain the even part of (24), which is given by

$$
\sum_{n=0}^{\infty} p_{\xi}(4 n) q^{2 n}=4 q^{4} \frac{f_{24}^{6}}{f_{6}^{2} f_{12}^{3}}-q^{4} \omega\left(-q^{6}\right)+\frac{f_{4}^{4} f_{12}^{5}}{f_{2}^{2} f_{6}^{4} f_{24}^{2}} .
$$

Replacing $q^{2}$ by $q$ we obtain (32).

Using (11) and (18) we can extract the odd part of (23):

$$
2 \sum_{n=0}^{\infty} p_{\xi}(4 n+1) q^{2 n+1}=4 q^{3} \frac{f_{12}^{3} f_{24}^{2}}{f_{6}^{4}}+4 q \frac{f_{8}^{4} f_{12}^{5}}{f_{4}^{2} f_{6}^{4} f_{24}^{2}} .
$$


After simplifications we arrive at (33).

Next, extracting the odd part of (24) with the help of (11) and (18) yields

$$
\sum_{n=0}^{\infty} p_{\xi}(4 n+2) q^{2 n+1}=q \frac{f_{12}^{9}}{f_{6}^{6} f_{24}^{2}}+q \frac{f_{4}^{10} f_{24}^{2}}{f_{2}^{4} f_{6}^{2} f_{8}^{4} f_{12}},
$$

which, after simplifications, gives us (34).

In order to obtain (35), we use (11) and (18) in (23) to extract its even part:

$$
2 \sum_{n=0}^{\infty} p_{\xi}(4 n+3) q^{2 n+2}=\frac{f_{12}^{15}}{f_{6}^{8} f_{24}^{6}}-f\left(q^{12}\right)+4 q^{2} \frac{f_{4}^{4} f_{24}^{2}}{f_{2}^{2} f_{6}^{2} f_{12}} .
$$

Replacing $q^{2}$ by $q$ in this identity, we obtain (35).

\section{Arithmetic properties of $p_{\xi}(n)$}

Our first observation provides a characterization of $p_{\xi}(3 n)(\bmod 4)$.

Theorem 4.1. For all $n \geq 0$, we have

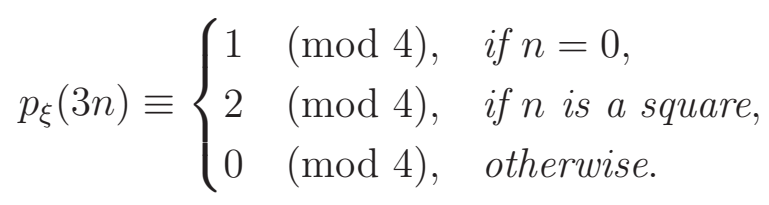

Proof. By (28), using (9) and the fact that $f_{k}^{4} \equiv f_{2 k}^{2}(\bmod 4)$ for all $k \geq 1$, it follows that

$$
\sum_{n=0}^{\infty} p_{\xi}(3 n) q^{n}=\frac{f_{2} f_{3}^{4}}{f_{1}^{2} f_{6}^{2}} \equiv \frac{f_{2}}{f_{1}^{2}}=\frac{f_{1}^{2} f_{2}}{f_{1}^{4}} \equiv \frac{f_{1}^{2}}{f_{2}}=\phi(-q) \quad(\bmod 4) .
$$

By (7), we obtain

$$
\sum_{n=0}^{\infty} p_{\xi}(3 n) q^{n} \equiv \sum_{n=-\infty}^{\infty}(-1)^{n} q^{n^{2}} \equiv 1+2 \sum_{n=1}^{\infty} q^{n^{2}} \quad(\bmod 4)
$$

which completes the proof. 
Theorem 4.1 yields an infinite family of Ramanujan-like congruences modulo 4 .

Corollary 4.1. For all primes $p>3$ and all $n \geq 0$, we have

$$
p_{\xi}(3(p n+r)) \equiv 0 \quad(\bmod 4)
$$

if $3 r$ is a quadratic nonresidue modulo $p$.

Proof. If $3(p n+r)=k^{2}$, then $3 r \equiv k^{2}(\bmod p)$, which contradicts the fact that $3 r$ is a quadratic nonresidue modulo $p$.

Since $\operatorname{gcd}(3, p)=1$, among the $p-1$ residues modulo $p$, we have $\frac{p-1}{2}$ residues $r$ for which $3 r$ is a quadratic nonresidue modulo $p$. Thus, for instance, the above corollary yields the following congruences:

$$
\begin{array}{ll}
p_{\xi}(15 n+k) \equiv 0 & (\bmod 4), \text { for } k \in\{3,12\}, \\
p_{\xi}(21 n+k) \equiv 0 & (\bmod 4), \text { for } k \in\{3,6,12\}, \\
p_{\xi}(33 n+k) \equiv 0 & (\bmod 4), \text { for } k \in\{6,18,21,24,30\} .
\end{array}
$$

Theorem 4.2. For all $n \geq 0$, we have

$$
p_{\xi}(3 n+1) \equiv\left\{\begin{array}{lll}
2 & (\bmod 4), & \text { if } 3 n+1 \text { is a square } \\
0 & (\bmod 4), & \text { otherwise }
\end{array}\right.
$$

Proof. From Theorem 3.2,

$$
\sum_{n=0}^{\infty} p_{\xi}(3 n+1) q^{n}=2 \frac{f_{3} f_{6}}{f_{1}}
$$

So we only need to consider the parity of

$$
\frac{f_{3} f_{6}}{f_{1}} .
$$

Note that

$$
\frac{f_{3} f_{6}}{f_{1}} \equiv \frac{f_{3}^{3}}{f_{1}}=\sum_{n=0}^{\infty} a_{3}(n) q^{n} \quad(\bmod 2)
$$


where $a_{3}(n)$ is the number of 3 -core partitions of $n$ (see [10, Theorem 1]). Thanks to [8, Theorem 7], we know that

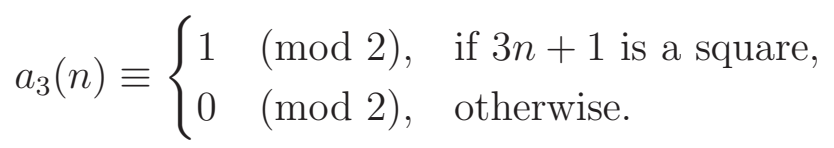

This completes the proof.

Theorem 4.2 yields an infinite family of congruences modulo 4 .

Corollary 4.2. For all primes $p>3$ and all $n \geq 0$, we have

$$
p_{\xi}(3(p n+r)+1) \equiv 0 \quad(\bmod 4),
$$

if $3 r+1$ is a quadratic nonresidue modulo $p$.

Proof. If $3(p n+r)+1=k^{2}$, then $3 r+1 \equiv k^{2}(\bmod p)$, which would be a contradiction with $3 r+1$ being a quadratic nonresidue modulo $p$.

For example, the following congruences hold for all $n \geq 0$ :

$$
\begin{array}{ll}
p_{\xi}(15 n+k) \equiv 0 & (\bmod 4), \text { for } k \in\{7,13\}, \\
p_{\xi}(21 n+k) \equiv 0 & (\bmod 4), \text { for } k \in\{10,13,19\}, \\
p_{\xi}(33 n+k) \equiv 0 & (\bmod 4), \text { for } k \in\{7,10,13,19,28\} .
\end{array}
$$

We next turn our attention to the arithmetic progression $4 n+2$ to yield an additional infinite family of congruences.

Theorem 4.3. For all $n \geq 0$, we have

$$
p_{\xi}(4 n+2) \equiv \begin{cases}(-1)^{k} \quad(\bmod 4), & \text { if } n=6 k(3 k-1), \\ 0 \quad(\bmod 4), & \text { otherwise. }\end{cases}
$$

Proof. From (34), we obtain

$$
\sum_{n=0}^{\infty} p_{\xi}(4 n+2) q^{n} \equiv \frac{f_{6}^{7}}{f_{3}^{2} f_{12}^{2}}+\frac{f_{12}^{2}}{f_{3}^{2} f_{6}} \equiv 2 \frac{f_{6}^{3}}{f_{3}^{2}} \equiv 2 f_{6}^{2} \equiv 2 f_{12} \quad(\bmod 4)
$$

Using Euler's identity (see [9, Eq. (1.6.1)])

$$
f_{1}=\sum_{n=-\infty}^{\infty}(-1)^{n} q^{n(3 n-1) / 2}
$$


we obtain

$$
\sum_{n=0}^{\infty} p_{\xi}(4 n+2) q^{n} \equiv \sum_{n=-\infty}^{\infty}(-1)^{n} q^{6 n(3 n-1)} \quad(\bmod 4)
$$

which concludes the proof.

Theorem 4.3 yields an infinite family of congruences modulo 4 .

Corollary 4.3. Let $p>3$ be a prime and $r$ an integer such that $2 r+1$ is a quadratic nonresidue modulo $p$. Then, for all $n \geq 0$,

$$
p_{\xi}(4(p n+r)+2) \equiv 0 \quad(\bmod 4) .
$$

Proof. If $p n+r=6 k(3 k-1)$, then $r \equiv 18 k^{2}-6 k(\bmod p)$. Thus, $2 r+1 \equiv$ $(6 k-1)^{2}(\bmod p)$, which contradicts the fact that $2 r+1$ is a quadratic nonresidue modulo $p$.

Thanks to Corollary 4.3, the following example congruences hold for all $n \geq 0$ :

$$
\begin{aligned}
& p_{\xi}(20 n+j) \equiv 0 \quad(\bmod 4), \quad \text { for } j \in\{6,14\} \text {, } \\
& p_{\xi}(28 n+j) \equiv 0 \quad(\bmod 4), \text { for } j \in\{6,10,26\} \text {, } \\
& p_{\xi}(44 n+j) \equiv 0 \quad(\bmod 4), \text { for } j \in\{14,26,34,38,42\} \text {, } \\
& p_{\xi}(52 n+j) \equiv 0 \quad(\bmod 4), \text { for } j \in\{10,14,22,30,38,42\} \text {. }
\end{aligned}
$$

We now provide a mod 8 characterization for $p_{\xi}(3 n)$.

Theorem 4.4. For all $n \geq 0$, we have

$$
p_{\xi}(3 n) \equiv \begin{cases}1 \quad(\bmod 8), & \text { if } n=0, \\ 6(-1)^{k}(\bmod 8), & \text { if } n=k^{2}, \\ 4 \quad(\bmod 8), & \text { if } n=2 k^{2}, n=3 k^{2}, \text { or } n=6 k^{2} \\ 0 \quad(\bmod 8), & \text { otherwise. }\end{cases}
$$


Proof. By (28), using (7) and (9), we have

$$
\begin{aligned}
\sum_{n=0}^{\infty} p_{\xi}(3 n) q^{n} & =\frac{f_{1}^{6} f_{2} f_{3}^{4}}{f_{1}^{8} f_{6}^{2}} \equiv\left(\frac{f_{1}^{2}}{f_{2}}\right)^{3}\left(\frac{f_{3}^{2}}{f_{6}}\right)^{2} \equiv \phi(-q)^{3} \phi\left(-q^{3}\right)^{2} \\
& \equiv\left(\sum_{n=-\infty}^{\infty}(-1)^{n} q^{n^{2}}\right)^{3}\left(\sum_{n=-\infty}^{\infty}(-1)^{n} q^{3 n^{2}}\right)^{2} \\
& \equiv\left(1+2 \sum_{n=1}^{\infty}(-1)^{n} q^{n^{2}}\right)^{3}\left(1+2 \sum_{n=1}^{\infty}(-1)^{n} q^{3 n^{2}}\right)^{2}(\bmod 8)
\end{aligned}
$$

which yields

$$
\begin{aligned}
\sum_{n=0}^{\infty} p_{\xi}(3 n) q^{n} \equiv & 1+6 \sum_{n=1}^{\infty}(-1)^{n} q^{n^{2}}+4\left(\sum_{n=1}^{\infty}(-1)^{n} q^{n^{2}}\right)^{2} \\
& +4 \sum_{n=1}^{\infty}(-1)^{n} q^{3 n^{2}}+4\left(\sum_{n=1}^{\infty}(-1)^{n} q^{3 n^{2}}\right)^{2} \quad(\bmod 8)
\end{aligned}
$$

Since

$$
\left(\sum_{n=1}^{\infty}(-1)^{n} q^{n^{2}}\right)^{2} \equiv \sum_{n=-\infty}^{\infty} q^{2 n^{2}} \quad(\bmod 2)
$$

we have

$$
\left(\sum_{n=1}^{\infty}(-1)^{n} q^{3 n^{2}}\right)^{2} \equiv \sum_{n=-\infty}^{\infty} q^{6 n^{2}} \quad(\bmod 2)
$$

Therefore

$$
\begin{aligned}
\sum_{n=0}^{\infty} p_{\xi}(3 n) q^{n} \equiv & 1+6 \sum_{n=1}^{\infty}(-1)^{n} q^{n^{2}}+4 \sum_{n=1}^{\infty} q^{2 n^{2}} \\
& +4 \sum_{n=1}^{\infty}(-1)^{n} q^{3 n^{2}}+4 \sum_{n=1}^{\infty} q^{6 n^{2}} \quad(\bmod 8)
\end{aligned}
$$

which completes the proof.

As with the prior results, Theorem 4.4 provides an effective way to yield an infinite family of congruences modulo 8 . 
Corollary 4.4. Let $p$ be a prime such that $p \equiv \pm 1(\bmod 24)$. Then,

$$
p_{\xi}(3(p n+r)) \equiv 0 \quad(\bmod 8)
$$

if $r$ is a quadratic nonresidue modulo $p$.

Proof. Since $p \equiv \pm 1(\bmod 8)$ and $p \equiv \pm 1(\bmod 12)$, it follows that 2 and 3 are quadratic residues modulo $p$. Thus, $r, 2 r, 3 r$, and $6 r$ are quadratic nonresidues modulo $p$. Indeed, according to the properties of Legendre's symbol, for $j \in\{1,2,3,6\}$, we have

$$
\left(\frac{j r}{p}\right)=\left(\frac{j}{p}\right)\left(\frac{r}{p}\right)=\left(\frac{r}{p}\right)=-1 .
$$

It follows that we cannot have $3(p n+r)=j k^{2}$, for some $k \in \mathbb{N}$ and $j \in$ $\{1,2,3,6\}$. In fact, $3(p n+r)=j k^{2}$ would imply $3(p n+r) \equiv 3 r \equiv j k^{2}$ $(\bmod p)$. However, for $j=1,2,3,6$, this would imply that $3 r, 6 r, r$, or $2 r$, respectively, is a quadratic residue modulo $p$, which would be a contradiction since 2,3 , and 6 are quadratic residues modulo $p$. The result follows from Theorem 4.4 .

As an example, we note that, for $p=23$ and all $n \geq 0$, we have $p_{\xi}(69 n+k) \equiv 0 \quad(\bmod 8)$, for $k \in\{15,21,30,33,42,45,51,57,60,63,66\}$.

Theorem 4.5. For all $n \geq 0$, we have

$$
p_{\xi}(12 n+4) \equiv p_{\xi}(3 n+1) \quad(\bmod 8) \text {. }
$$

Proof. Initially we use (14) to extract the odd part on both sides of (29). The resulting identity is

$$
\sum_{n=0}^{\infty} p_{\xi}(6 n+4) q^{n}=2 \frac{f_{3}^{2} f_{4}^{2} f_{24}}{f_{1}^{2} f_{8} f_{12}}
$$

Using (15) in (38), we obtain

$$
\sum_{n=0}^{\infty} p_{\xi}(12 n+4) q^{n}=2 \frac{f_{2}^{6} f_{3} f_{6}}{f_{1}^{5} f_{4}^{2}} \equiv 2 \frac{f_{1}^{3} f_{2}^{6} f_{3} f_{6}}{f_{1}^{8} f_{4}^{2}} \equiv 2 \frac{f_{3} f_{6}}{f_{1}} \quad(\bmod 8) .
$$

The result follows using (29). 
Now we present complete characterizations of $p_{\xi}(48 n+4)$ and $p_{\xi}(12 n+1)$ modulo 8.

Theorem 4.6. For all $n \geq 0$, we have

$$
p_{\xi}(48 n+4) \equiv p_{\xi}(12 n+1) \equiv \begin{cases}2(-1)^{k} \quad(\bmod 8), & \text { if } n=k(3 k-1), \\ 0 \quad(\bmod 8), & \text { otherwise }\end{cases}
$$

Proof. The first congruence follows directly from Theorem 4.5. Replacing (14) in (29), we obtain

$$
\sum_{n=0}^{\infty} p_{\xi}(3 n+1) q^{n}=2 \frac{f_{4} f_{6}^{2} f_{16} f_{24}^{2}}{f_{2}^{2} f_{8} f_{12} f_{48}}+2 q \frac{f_{6}^{2} f_{8}^{2} f_{48}}{f_{2}^{2} f_{16} f_{24}} .
$$

Extracting the terms of the form $q^{2 n}$, we have

$$
\sum_{n=0}^{\infty} p_{\xi}(6 n+1) q^{2 n}=2 \frac{f_{4} f_{6}^{2} f_{16} f_{24}^{2}}{f_{2}^{2} f_{8} f_{12} f_{48}},
$$

which, after replacing $q^{2}$ by $q$, yields

$$
\sum_{n=0}^{\infty} p_{\xi}(6 n+1) q^{n}=2 \frac{f_{2} f_{3}^{2} f_{8} f_{12}^{2}}{f_{1}^{2} f_{4} f_{6} f_{24}}
$$

Now we use (15) to obtain

$$
\begin{aligned}
\sum_{n=0}^{\infty} p_{\xi}(12 n+1) q^{n} & =2 \frac{f_{2}^{3} f_{6}^{4}}{f_{1}^{4} f_{12}^{2}} \\
& \equiv 2 f_{2} \equiv 2 \sum_{n=-\infty}^{\infty}(-1)^{n} q^{n(3 n-1)} \quad(\bmod 8)
\end{aligned}
$$

which completes the proof.

Theorem 4.6 also provides an effective way to yield an infinite family of congruences modulo 8.

Corollary 4.5. For all primes $p>3$ and all $n \geq 0$, we have

$$
p_{\xi}(48(p n+r)+4) \equiv p_{\xi}(12(p n+r)+1) \equiv 0 \quad(\bmod 8),
$$

if $12 r+1$ is a quadratic nonresidue modulo $p$.

Proof. Let $p>3$ be a prime and $12 r+1$ a quadratic nonresidue modulo $p$. If $p n+r=k(3 k-1)$, then $r \equiv 3 k^{2}-k(\bmod p)$, which implies that $12 r+1 \equiv$ $(6 k-1)^{2}(\bmod p)$, a contradiction. The result follows from Theorem 4.6. 


\section{Additional congruences}

In this section, we prove several additional Ramanujan-like congruences that are not included in the results of the previous section.

Theorem 5.1. For all $n \geq 0$, we have

$$
\begin{array}{ll}
p_{\xi}(24 n+19) \equiv 0 & (\bmod 3), \\
p_{\xi}(27 n+18) \equiv 0 & (\bmod 3), \quad \text { and } \\
p_{\xi}(72 n+51) \equiv 0 & (\bmod 3) .
\end{array}
$$

Proof. Using (15) we can now 2-dissect (39) to obtain

$$
\sum_{n=0}^{\infty} p_{\xi}(6 n+1) q^{n}=2 \frac{f_{4}^{3} f_{12}^{4}}{f_{1}^{2} f_{2}^{4} f_{24}^{2}}+4 q \frac{f_{6} f_{8}^{2} f_{12}}{f_{2}^{3}}
$$

from which we have

$$
\sum_{n=0}^{\infty} p_{\xi}(12 n+7) q^{2 n+1}=4 q \frac{f_{6} f_{8}^{2} f_{12}}{f_{2}^{3}} .
$$

Now, dividing both sides of the above expression by $q$ and replacing $q^{2}$ by $q$, we obtain

$$
\sum_{n=0}^{\infty} p_{\xi}(12 n+7) q^{n}=4 \frac{f_{3} f_{4}^{2} f_{6}}{f_{1}^{3}} .
$$

Using (17) we rewrite (43) as

$$
\sum_{n=0}^{\infty} p_{\xi}(12 n+7) q^{n}=4 \frac{f_{4}^{8} f_{6}^{4}}{f_{2}^{9} f_{12}^{2}}+12 q \frac{f_{4}^{4} f_{6}^{2} f_{12}^{2}}{f_{2}^{7}}
$$

Taking the odd parts on both sides of the last equation, we are left with

$$
\sum_{n=0}^{\infty} p_{\xi}(24 n+19) q^{n}=12 \frac{f_{2}^{4} f_{3}^{2} f_{6}^{2}}{f_{1}^{7}}
$$

which proves (40). 
In order to prove (41), we use (22) to extract the terms of the form $q^{3 n}$ of (28). The resulting identity is

$$
\sum_{n=0}^{\infty} p_{\xi}(9 n) q^{3 n}=\frac{f_{6}^{2} f_{9}^{6}}{f_{3}^{4} f_{18}^{3}}
$$

which, after replacing $q^{3}$ by $q$ and using (8), yields

$$
\sum_{n=0}^{\infty} p_{\xi}(9 n) q^{n}=\frac{f_{2}^{2} f_{3}^{6}}{f_{1}^{4} f_{6}^{3}} \equiv \frac{f_{2}^{2} f_{3}^{5}}{f_{1} f_{6}^{3}}=\psi(q) \frac{f_{3}^{5}}{f_{6}^{3}} \quad(\bmod 3) .
$$

By (8), we have

$$
\sum_{n=0}^{\infty} p_{\xi}(9 n) q^{n} \equiv \frac{f_{3}^{5}}{f_{6}^{3}} \sum_{n=0}^{\infty} q^{n(n+1) / 2} \quad(\bmod 3) .
$$

Since $n(n+1) / 2 \not \equiv 2(\bmod 3)$ for all $n \geq 0$, all terms of the form $q^{3 n+2}$ in the last expression have coefficients congruent to $0(\bmod 3)$, which proves $(41)$.

We now prove (42). Replacing (22) in (28) and extracting the terms of the form $q^{3 n+2}$, we obtain

$$
\sum_{n=0}^{\infty} p_{\xi}(9 n+6) q^{3 n+2}=4 q^{2} \frac{f_{18}^{3}}{f_{3}^{2}}
$$

Dividing both sides of (44) by $q^{2}$ and replacing $q^{3}$ by $q$, we have

$$
\sum_{n=0}^{\infty} p_{\xi}(9 n+6) q^{n}=4 \frac{f_{6}^{3}}{f_{1}^{2}}
$$

Now we use (11) to extract the odd part of (45) and obtain

$$
\sum_{n=0}^{\infty} p_{\xi}(18 n+15) q^{n}=8 \frac{f_{2}^{2} f_{3}^{3} f_{8}^{2}}{f_{1}^{5} f_{4}}
$$

Since $f_{1}^{3} \equiv f_{3}(\bmod 3)$, we have

$$
\sum_{n=0}^{\infty} p_{\xi}(18 n+15) q^{n} \equiv 2 \frac{f_{2}^{2} f_{3}^{2} f_{8}^{2}}{f_{1}^{2} f_{4}} \quad(\bmod 3) .
$$


Using (15) we obtain

$$
\sum_{n=0}^{\infty} p_{\xi}(36 n+15) q^{n} \equiv 2 \frac{f_{2}^{3} f_{3} f_{4} f_{6}^{2}}{f_{1}^{3} f_{12}} \quad(\bmod 3) .
$$

Since the odd part of $(17)$ is divisible by 3 , then the coefficients of the terms of the form $q^{2 n+1}$ in $\sum_{n=0}^{\infty} p_{\xi}(36 n+15) q^{n}$ are congruent to 0 modulo 3 . This completes the proof of (42).

Theorem 5.2. For all $n \geq 0$, we have

$$
\begin{aligned}
p_{\xi}(8 n+6) & \equiv 0 \quad(\bmod 4), \\
p_{\xi}(16 n+10) & \equiv 0 \quad(\bmod 4) .
\end{aligned}
$$

Proof. Congruence (46) follows directly by extracting the odd part of (37). Extracting the even part of (37) yields

$$
\sum_{n=0}^{\infty} p_{\xi}(8 n+2) q^{n} \equiv 2 f_{6} \quad(\bmod 4)
$$

from which (47) follows.

We now prove a pair of unexpected congruences modulo 5 satisfied by $p_{\xi}(n)$.

Theorem 5.3. For all $n \geq 0$, we have

$$
\begin{array}{ll}
p_{\xi}(45 n+33) \equiv 0 & (\bmod 5), \\
p_{\xi}(45 n+41) \equiv 0 & (\bmod 5) .
\end{array}
$$

Proof. By (45), we have

$$
\sum_{n=0}^{\infty} p_{\xi}(9 n+6) q^{n}=4 \frac{f_{6}^{3}}{f_{1}^{2}}=4 \frac{f_{1}^{3} f_{6}^{3}}{f_{1}^{5}} \equiv 4 \frac{f_{1}^{3} f_{6}^{3}}{f_{5}} \quad(\bmod 5) .
$$

Thanks to Jacobi's identity (10) we know

$$
f_{1}^{3} f_{6}^{3}=\sum_{j, k=0}^{\infty}(-1)^{j+k}(2 j+1)(2 k+1) q^{3 j(j+1)+k(k+1) / 2} .
$$

Note that, for all integers $j$ and $k, 3 j(j+1)$ and $k(k+1) / 2$ are congruent to either 0,1 or 3 modulo 5 . The only way to obtain $3 j(j+1)+k(k+1) / 2=5 n+3$ is the following: 
- $3 j(j+1) \equiv 0(\bmod 5)$ and $k(k+1) / 2 \equiv 3(\bmod 5)$, or

- $3 j(j+1) \equiv 3(\bmod 5)$ and $k(k+1) / 2 \equiv 0(\bmod 5)$.

Thus, $j \equiv 2(\bmod 5)$ or $k \equiv 2(\bmod 5)$ in all possible cases, and this means

$$
(2 j+1)(2 k+1) \equiv 0 \quad(\bmod 5) .
$$

Therefore, for all $n \geq 0, p_{\xi}(45 n+33)=p_{\xi}(9(5 n+3)+6) \equiv 0(\bmod 5)$, which is (48).

In order to complete the proof of (49), we want to see when

$$
3 j(j+1)+k(k+1) / 2=5 n+4 .
$$

Four possible cases arise:

- $k \equiv 1(\bmod 5)$ and $j \equiv 2(\bmod 5)$,

- $k \equiv 3(\bmod 5)$ and $j \equiv 2(\bmod 5)$,

- $j \equiv 1(\bmod 5)$ and $k \equiv 2(\bmod 5), \quad$ or

- $j \equiv 3(\bmod 5)$ and $k \equiv 2(\bmod 5)$.

In all four cases above, either $j \equiv 2(\bmod 5)$ or $k \equiv 2(\bmod 5)$. So

$$
(2 j+1)(2 k+1) \equiv 0 \quad(\bmod 5)
$$

in all these cases. Therefore,

$$
p_{\xi}(45 n+42)=p_{\xi}(9(5 n+4)+6) \equiv 0 \quad(\bmod 5),
$$

which completes the proof of (49).

Next, we prove three congruences modulo 8 which are not covered by the above results.

Theorem 5.4. For all $n \geq 0$, we have

$$
\begin{array}{ll}
p_{\xi}(16 n+14) \equiv 0 & (\bmod 8), \\
p_{\xi}(24 n+13) \equiv 0 & (\bmod 8), \\
p_{\xi}(24 n+22) \equiv 0 & (\bmod 8) .
\end{array}
$$


Proof. Initially we prove (50). From (34) and (7) we have

$$
\sum_{n=0}^{\infty} p_{\xi}(4 n+2) q^{n} \equiv \frac{f_{3}^{2} f_{6}^{5}}{f_{12}^{2}}+\frac{f_{12}^{2}}{f_{3}^{2} f_{6}} \phi(q)^{2} \quad(\bmod 8) \text {. }
$$

Now we can use (11), (12), and (20) to extract the terms involving $q^{2 n+1}$ from both sides of the previous congruence:

$$
\sum_{n=0}^{\infty} p_{\xi}(8 n+6) q^{2 n+1} \equiv-2 q^{3} \frac{f_{6}^{6} f_{48}^{2}}{f_{12}^{2} f_{24}}+2 q^{3} \frac{f_{4}^{10} f_{12}^{4} f_{48}^{2}}{f_{2}^{4} f_{6}^{6} f_{8}^{4} f_{24}}+4 q \frac{f_{8}^{4} f_{12}^{2} f_{24}^{5}}{f_{4}^{2} f_{6}^{6} f_{48}^{2}} \quad(\bmod 8) .
$$

After dividing both sides by $q$ and then replacing $q^{2}$ by $q$, we are left with

$$
\begin{aligned}
\sum_{n=0}^{\infty} p_{\xi}(8 n+6) q^{n} & \equiv-2 q \frac{f_{3}^{6} f_{24}^{2}}{f_{6}^{2} f_{12}}+2 q \frac{f_{2}^{10} f_{6}^{4} f_{24}^{2}}{f_{1}^{4} f_{3}^{6} f_{4}^{4} f_{12}}+4 \frac{f_{4}^{4} f_{6}^{2} f_{12}^{5}}{f_{2}^{2} f_{3}^{6} f_{24}^{2}} \\
& \equiv-2 q \frac{f_{3}^{6} f_{24}^{2}}{f_{6}^{2} f_{12}}+2 q \frac{f_{3}^{6} f_{6}^{4} f_{24}^{2}}{f_{3}^{12} f_{12}}+4 \frac{f_{4}^{4} f_{12}^{5}}{f_{2}^{2} f_{6} f_{24}^{2}} \\
& \equiv 4 \frac{f_{4}^{3} f_{12}}{f_{6}}(\bmod 8),
\end{aligned}
$$

whose odd part is congruent to 0 modulo 8, which implies (50).

In order to prove (51), we use (15) to obtain the even part of identity (39), which is

$$
\sum_{n=0}^{\infty} p_{\xi}(12 n+1) q^{n}=2 \frac{f_{2}^{3} f_{6}^{4}}{f_{1}^{4} f_{12}^{2}}
$$

Now, employing (13), we obtain the odd part of the last identity, which is

$$
\sum_{n=0}^{\infty} p_{\xi}(24 n+13) q^{n}=8 \frac{f_{2}^{2} f_{3}^{4} f_{4}^{4}}{f_{1}^{7} f_{6}^{2}}
$$

which implies (51).

Now we prove (52). We employ (15) in (38) to obtain

$$
\sum_{n=0}^{\infty} p_{\xi}(12 n+10) q^{n}=4 \frac{f_{2}^{3} f_{3}^{2} f_{12}^{2}}{f_{1}^{4} f_{6}}
$$


By (12) and (13), we rewrite (53) in the form

$$
\sum_{n=0}^{\infty} p_{\xi}(12 n+10) q^{n}=4 \frac{f_{2}^{3} f_{12}^{2}}{f_{6}^{2}}\left(\frac{f_{4}^{14}}{f_{2}^{14} f_{8}^{4}}+4 q \frac{f_{4}^{2} f_{8}^{4}}{f_{2}^{10}}\right)\left(\frac{f_{6} f_{24}^{5}}{f_{12}^{2} f_{48}^{2}}-2 q^{3} \frac{f_{6} f_{48}^{2}}{f_{2} 4}\right)
$$

from which we obtain

$$
\sum_{n=0}^{\infty} p_{\xi}(24 n+22) q^{2 n+1}=4 \frac{f_{2}^{3} f_{12}^{2}}{f_{6}^{2}}\left(-2 q^{3} \frac{f_{4}^{14} f_{6} f_{48}^{2}}{f_{2}^{14} f_{8}^{4} f_{24}}+4 q \frac{f_{4}^{2} f_{6} f_{8}^{4} f_{24}^{5}}{f_{2}^{10} f_{12}^{2} f_{48}^{2}}\right) .
$$

Dividing both sides by $q$ and replacing $q^{2}$ by $q$, we are left with

$$
\sum_{n=0}^{\infty} p_{\xi}(24 n+22) q^{n}=-8 q \frac{f_{2}^{14} f_{6}^{2} f_{24}^{2}}{f_{1}^{11} f_{3} f_{4}^{4} f_{12}}+16 \frac{f_{2}^{2} f_{4}^{4} f_{12}^{5}}{f_{1}^{7} f_{3} f_{24}^{2}}
$$

which implies (52).

We close this section by proving a congruence modulo 9 .

Theorem 5.5. For all $n \geq 0$, we have

$$
p_{\xi}(96 n+76) \equiv 0 \quad(\bmod 9) .
$$

Proof. We use (21) to extract the terms of the form $q^{3 n+1}$ from (32). The resulting identity is

$$
\sum_{n=0}^{\infty} p_{\xi}(12 n+4) q^{3 n+1}=2 q \frac{f_{6}^{6} f_{9} f_{18}}{f_{3}^{5} f_{12}^{2}}
$$

which, after dividing by $q$ and replacing $q^{3}$ by $q$, yields

$$
\sum_{n=0}^{\infty} p_{\xi}(12 n+4) q^{n}=2 \frac{f_{2}^{6} f_{3} f_{6}}{f_{1}^{5} f_{4}^{2}}=2 \frac{f_{2}^{6} f_{6}}{f_{4}^{2}} \frac{f_{3}}{f_{1}} \frac{1}{f_{1}^{4}} .
$$

Using (13) and (14), we extract the even part on both sides of the above identity to obtain

$$
\begin{aligned}
\sum_{n=0}^{\infty} p_{\xi}(24 n+4) q^{n} & =2 \frac{f_{2}^{13} f_{3}^{2} f_{8} f_{12}^{2}}{f_{1}^{10} f_{4}^{5} f_{6} f_{24}}+8 q \frac{f_{3}^{2} f_{4}^{6} f_{24}}{f_{1}^{6} f_{8} f_{12}} \\
& \equiv 2 \frac{f_{2}^{13} f_{8} f_{12}^{2}}{f_{4}^{5} f_{6} f_{24}} \frac{1}{f_{1} f_{3}}+8 q \frac{f_{4}^{6} f_{24}}{f_{8} f_{12}} \frac{f_{1}^{3}}{f_{3}} \quad(\bmod 9) .
\end{aligned}
$$


Now we employ (18) and (16) to extract the odd part on both sides of the last congruence:

$$
\sum_{n=0}^{\infty} p_{\xi}(48 n+28) q^{n} \equiv 2 \frac{f_{1}^{9} f_{6} f_{12}}{f_{3}^{3} f_{4}}+8 \frac{f_{2}^{9} f_{12}}{f_{4} f_{6}^{2}} \equiv \frac{f_{6} f_{12}}{f_{4}} \quad(\bmod 9),
$$

which implies (54).

\section{Concluding remarks}

Computational evidence indicates that $p_{\xi}(n)$ satisfies many other congruences. The interested reader may wish to consider the following two conjectures.

\section{Conjecture 6.1.}

$$
\sum_{n=0}^{\infty} p_{\xi}(8 n+3) q^{n} \equiv 2 \sum_{n=0}^{\infty} q^{3 n(n+1) / 2} \quad(\bmod 3)
$$

\section{Conjecture 6.2.}

$$
\sum_{n=0}^{\infty} p_{\xi}(32 n+12) q^{n} \equiv 6 \sum_{n=0}^{\infty} q^{3 n(n+1) / 2} \quad(\bmod 9)
$$

Clearly, once proven, Conjectures 6.1 and 6.2 would immediately lead to infinite families of Ramanujan-like congruences. Morever, Conjecture 6.2 would immediately imply Theorem 5.5 since $96 n+76=32(3 n+2)+12$ while the right-hand side of Conjecture 6.2 is clearly a function of $q^{3}$. The same argument would imply that, for all $n \geq 0$,

$$
p_{\xi}(96 n+44) \equiv 0 \quad(\bmod 9)
$$

since $96 n+44=32(3 n+1)+12$.

\section{Acknowledgment}

The first author was supported by São Paulo Research Foundation (FAPESP) (grant no. 2019/14796-8). 


\section{References}

[1] G.E. Andrews, A. Dixit, A. J. Yee, Partitions associated with the Ramanujan/Watson mock theta functions $\omega(q)$ and $\phi(q)$, Res. Number Theory 1 (2015) 1-19.

[2] G. E. Andrews, D. Passary, J. A. Sellers, and A. J. Yee, Congruences related to the Ramanujan/Watson mock theta functions $\omega(q)$ and $\nu(q)$, Ramanujan J. 43 (2017), 347-357.

[3] B. C. Berndt, Ramanujan's Notebooks, Part III. Springer, New York, 1991.

[4] B. C. Berndt, Number Theory in the Spirit of Ramanujan. American Mathematical Society, 2006.

[5] E. H. M. Brietzke, R. da Silva, and J. A. Sellers, Congruences related to an eighth order mock theta function of Gordon and McIntosh, J. Math. Anal. Appl. 479 (2019), 62-89.

[6] B. Gordon and R. J. McIntosh, Some eight order mock theta functions, J. Lond. Math. Soc. 62 (2000), 321-335.

[7] B. Gordon and R. J. McIntosh, Modular transformations of Ramanujan's fifth and seventh order mock theta functions. Ramanujan J. 7 (2003), 193-222.

[8] R. da Silva and J. A. Sellers, Parity Considerations for the Mex-Related Partition Functions of Andrews and Newman, J. Integer Seq. 23 (2020), Article 20.5.7.

[9] M. D. Hirschhorn, The power of $q$, a personal journey, Developments in Mathematics, v. 49, Springer, 2017.

[10] M. D. Hirschhorn and J. A. Sellers, Elementary proofs of various facts about 3-cores, Bull. Aust. Math. Soc. 79 (2009), 507512.

[11] M. D. Hirschhorn and J. A. Sellers, Arithmetic relations for overpartitions, J. Combin. Math. Combin. Comput. 53 (2005) 65-73.

[12] R. J. McIntosh, New mock theta conjectures Part I, Ramanujan J. 46 (2018), 593-604. 
[13] M. S. M. Naika and D. S. Gireesh, Congruences for 3-regular partitions with designated summands. Integers 16 (2016), A25.

[14] L. Wang, New congruences for partitions related to mock theta functions, J. Number Theory 175 (2017) 51-65.

[15] W. Zhang and J. Shi, Congruences for the coefficients of the mock theta function $\beta(q)$. Ramanujan J. 49 (2019), 257-267.

Universidade Federal de São Paulo, Av. Cesare M. G. Lattes, 1201, São José dos Campos, SP, 12247-014, Brazil.

E-mail address: silva.robson@unifesp.br

Department of Mathematics and Statistics, University of Minnesota Duluth, Duluth, MN 55812, USA.

E-mail address: jsellers@d.umn.edu 